\title{
IS SONIFICATION DOOMED TO FAIL?
}

\author{
John G. Neuhoff \\ The College of Wooster, \\ Wooster, OH 44691, USA \\ jneuhoff@wooster.edu
}

\begin{abstract}
Despite persistent research and design efforts over the last twenty years, widespread adoption of sonification to display complex data has largely failed to materialize, and many of the challenges to successful sonification identified in the past persist. Major impediments to the widespread adoption sonification include fundamental perceptual differences between vision and audition, large individual differences in auditory perception, musical biases of sonification researchers, and the interdisciplinary nature of sonification research and design. The historical and often indiscriminate mingling of art and science in sonification design may be a root cause of some of these challenges. Future sonification design efforts that explicitly strive to meet either artistic or scientific goals may lead to greater clarity and success in the field and more widespread adoption of useful sonification techniques.
\end{abstract}

\section{INTRODUCTION}

This year marks the $20^{\text {th }}$ anniversary of the publication of The Sonification Report [1]. An international panel of sonification researchers produced the report which identified the state of the field at that time and a research agenda going forward. In the time since the report, sonification has grown slowly but steadily. A March 2019 search of all Web of Science databases showed a nearly four-fold increase in the appearance of the term "sonification" in literature over the previous 20 years. However, despite some innovative one-off successes, the widespread adoption of sonification to present complex data has largely failed to materialize. In fact, most sonifications in widespread use today are simple binary messages (e.g., ding! your seatbelt is unlatched).

However, researchers have been anticipating a tipping point in the field for some time. The authors of The Sonification Report in 1999 wrote that "Sonification will gain significant momentum once several specific applications become widely used. However, until there are intuitive, efficacious applications, skeptics will adhere to current display solutions." Twenty years later the quest for success and the "intuitive, efficacious application" or the killer app, as it came to be known, continues [2,3].

\section{HOW SHOULD SUCCESS BE DEFINED?}

Is the killer app the appropriate metric by which we should measure the success of sonification? Should researchers continue to strive to make data sonification as ubiquitous a means of data representation as the bar graph? Some would say no. Nees [2] for example, argues that sonification is simply one kind of tool that can be used to display data. He cites several successful (if not ubiquitous) examples where sonification (c) (1) (s)

This work is licensed under Creative Commons Attribution - Non Commercial 4.0 International License.

The full terms of the License are available at

http://creativecommons.org/licenses/by-nc/4.0/

https://doi.org/10.21785/icad2019.069 "works." Nees argues that if sonification in the appropriate context conveys the intended information, then the field as a whole can be considered successful. However, even those who promote most strongly the viability of widespread sonification and argue that a killer app is not required for success acknowledge that many of the roadblocks to successful sonification identified in The Sonification Report by Kramer et al. in 1999 are still prevalent today [2, 4-6].

\section{PERSISTENT CHALLENGES TO SONIFICATION}

The quest for sonification success has yielded several different approaches to representing data with sound. Audification, auditory icons, earcons, parameter mapping, and model-based sonification all have strengths and weaknesses. Their collective promise has led to somewhat of a public fascination with the idea of sonification and a relentless sense of optimism within the sonification research community [7, 8]. Unfortunately, sonification is more often viewed by the public as an entertaining curiosity than as a scientific tool for understanding data [9]. Some of the reasons for this include fundamental perceptual differences between vision and audition, large individual differences in auditory perception, perceptual crosstalk in audition, inherent musical biases of sonification researchers, and the interdisciplinary nature of the field.

The precision of vision versus audition. In humans, there are approximately ten times as many cortical neurons devoted to vision as there are to hearing. It should come as no surprise then that in all but the perception of time, perceptual judgments made with the eyes are usually more precise than those made with the ears. For example, the most common representational dimension used in visual graphs is length. The most commonly used dimension in auditory graphs is pitch [10]. If we examine the just noticeable difference (the minimum amount that a stimulus needs to change in order for the observer to notice the change) in each dimension, we find the percentage of pitch change required to notice a change is about twice the percentage of line length change required $[11,12]$. If we examine the spatial resolution of the two modalities we find that the auditory system has a resolution or Minimum Audible Angle of between one and two degrees azimuth [13]. The corresponding visual measure, the Minimum Angle of Resolution is about 60 times more precise with a resolution of 1-2 minutes of arc [14]. In almost all dimensions but time, the precision with which we can perceptualize data is greater in vision. This disparity obviously presents some difficulty for making a sonification that is on par with a typical visualization.

Individual differences in audition. In addition to differences in precision, the polarity of mapping data to an auditory representation is more unreliable than mapping data to visual representation. For example, in a visual graph, "up" almost always represents "more." However, the same cannot be said for sonification. When data variables such as physical size or number of dollars are mapped to pitch, listeners are almost 
evenly split on the question as to whether increasing pitch should represent increasing or decreasing values of the variable in question. Other variables show similar individual differences $[5,15,16]$. Some listeners with little to no musical experience even show a poor grasp of what the words "up" and "down" mean in the context of pitch change [17].

Widespread individual differences in music cognition further compound these problems. For example, musicians have lower thresholds for pitch discrimination than nonmusicians [18], show enhanced attentive processing of nonspeech sounds [19], and demonstrate better acuity in pitch and time [20]. Perhaps the most critical difference between musicians and non-musicians in extracting information from sonification lies in the ability to segregate auditory streams. Extracting information about any one variable from a display requires selectively attending to the variable of interest and suppressing attention to the other simultaneously sounding streams, a task at which musically experienced listeners excel and novices struggle [21]. Thus, variability in music cognition leads to variability in the comprehension of most sonifications.

Auditory perceptual interaction and asymmetry. Compounding the problem of individual differences is the finding that many auditory perceptual dimensions that are used to represent multidimensional data have been shown to interact perceptually [22]. Changes in loudness can influence perceived changes in other dimensions such as pitch or timbre [23]. This type of interaction can distort the underlying relationships between the data variables. Complicating matters even further are findings that show increases in acoustic dimensions such as pitch, loudness, and tempo are perceived as changing more than identical decreases in those dimensions [24-26]. Thus, a data variable mapped to one of these dimensions that exhibits an increase of ten units would be heard as changing more than if the same variable decreased by ten units.

The musical nature of sonification researchers. There are 1,103 conference papers in the ICAD proceedings from the years 1994-2018. The word music appears in $74 \%$ of these papers, and musical terminology is used widely [3]. Over $30 \%$ of the authors listed on the 1,103 conference papers have an institutional or departmental affiliation related to music (e.g., School of Music). In addition to those whose primary employment is in the field of music, a large percentage of sonification researchers in other fields also have some background in music. There is typically a higher proportion of musicians among those who do research in audition as can be evidenced by both the programmatic and impromptu "jam sessions" that occur at among attendees at professional conferences such as the Meeting of the Acoustical Society of America, The Society for Music Perception \& Cognition, and $I C A D$. Among psychologists who study music cognition, over $97 \%$ report having a musical background [27].

The overrepresentation of musicians in the sonification community coupled with the dramatic differences between the brains and perceptual abilities of musicians and non-musicians has the potential to skew sonification design in a way that is not aligned with the listening practices and abilities of the general public [28]. Musicians employ analytical listening strategies that can be beyond the immediate grasp of non-musicians [29]. Importantly, the analytical listening advantage that musicians have is present even when listening to non-musical audio [30].

While some researchers have stressed the importance of taking individual differences like musical background into account from the start when designing a sonification [31], others have suggested that sonification designers "use their own introspection and intuition" in sonification design before moving to more formal usability testing [32]. Still, others have eschewed musically naïve listeners entirely and focused exclusively on those with domain expertise [33]. Thus, a major stumbling block to effective sonification design for the masses is a failure of designers to take the perspective of musically naïve and "non-attentive listeners" [34].

Interdisciplinarity. Sonification is an inherently interdisciplinary field. Economist George Steigler once said, "The main insight learned from interdisciplinary studies is the return to specialization." Challenges to interdisciplinary work include differences in the underlying assumptions of the various disciplines, differences vocabulary, methods, and in values among many others. Perhaps nowhere is this more apparent in sonification work than when it comes to the evaluation of sonification. Should the sonification be evaluated simply by the designer? By process of iterative participatory design? Or by tests of statistical significance with appropriate sample size? The answer generally depends on the discipline of the person answering the question. Is sonification art, design, science, or a mixture of all three?

\section{THE BIFURCATION OF SONIFICATION}

It may be that bifurcating sonification into well-defined paths of art and science would lead to greater success. The paths need not be mutually exclusive and would be most effective if pursued simultaneously. There are advantages to both.

Shift Toward Artistic Sonification. Given the challenges to sonifying data in a manner that stays empirically faithful to the underlying data, perhaps some researchers should abandon this pursuit altogether. Instead, "artistic sonification" would embrace the more aesthetic aspects of sonic representation, giving listeners a "sense" of the underlying data while perhaps not always perfectly preserving the underlying data relations. Barrass [35] has suggested this approach as prioritizing "usefulness" in design even if it means sacrificing a veridical understanding of the underlying data.

This technique might be considered analogous to a courtroom sketch artist who makes drawings of the key figures at a trial. The representation is certainly not a "precise" representation of the courtroom scene, yet it does convey information to the viewer in a way that is "useful." In fact, the creativity of the artist might even provide a better representation of the mood of the courtroom than a still photograph. This artistic approach would facilitate multiple interpretations of the same data [36]. The shift in focus might also enhance the role that sonification plays in generating enthusiasm for science both with the public. For example, Ballora [7] has suggested that despite concerted efforts to sonify data empirically, "sonification's potential value, like much of the scientific visualisation content, probably lies less in hard facts and more in how it may serve as a stimulant for curiosity."

Shift Toward Empirical Sonification. Others in the field might pivot more toward the empirical. If nothing else, the last twenty years of sonification research have clarified what does not work [4]. If sonification is to be considered a scientifically legitimate way of representing data, we should heed the lessons of the past. Specifically, the following points should be emphasized:

1. Design efforts should be focused in a perceptual space where audition performs well and individual differences are smallest. 
2. To avoid perceptual interactions, parameter mapping that uses simple acoustic dimensions like pitch and loudness should be largely abandoned.

3. Empirical sonification researchers should evaluate their designs with a focus on the poorest rather than the best analytical listeners in their target user population.

Leveraging audition's temporal advantage would likely be a more fruitful approach than concentrating on other perceptual dimensions (e.g., pitch and loudness). Similarly, although the spatial resolution of the visual system is better than that of the auditory system, we can only see a limited field of vision while we can hear in 360 degrees. Concentrating on design efforts that exploit these kinds of advantages is likely to produce significant advances in the field. Abandoning the use of simple acoustic dimensions in parameter mapping would also be a step in the right direction. It has been known for decades that representing multidimensional data with multiple acoustic dimensions introduces distortions [23, 37, 38]. However, many current attempts to sonify data still take this approach [39-41]. As an alternative, ecological parameter mapping techniques might provide a more effective approach. The well-known work on ecological acoustics by Gaver [42] suggests that people listen to sounding objects and events rather than acoustic dimensions. As such, a better approach to parameter mapping might be assigning data variables to acoustically complex but ecologically simple sounds (e.g., footsteps) that indicate changes in sounding objects or events [43].

Finally, the importance of perspective taking by musically experienced sonification designers cannot be overstated. It is well known that musicians hear, think about, and speak about sound differently than non-musicians. Musicologist Sarah Cassie Provost gives her music students an assignment entitled "Communicating with Non-Musicians" [44]. Others provide "translations" for musicians who may find themselves working with non-musicians in a professional production environment [45]. A sonification designed by someone with a musical background could be largely lost on someone without one. An empirical approach to sonification would benefit from a design process that seeks input at the start from non-musicians and is evaluated empirically with a representative target population.

Avoid the "muddled middle." The line that separates art and science in sonification design is, in fact, not a line at all. Integrating art and science in sonification work has resulted in a continuum. Unfortunately, the closer a given sonification is to the midpoint, the more frequently it fails to live up to the goals of either art or science. Shifting sonification closer to the endpoints of this continuum would result in moving away from the muddled middle ground. Artistic sonification would have the goal of aesthetically enhancing user experience, capturing attention, and stimulating curiosity. It would be data-based without requiring an isomorphic tie between data and sound. Scientific sonification would have the goal of reliably representing the underlying data across listening conditions and listeners. It would not ignore aesthetics but would hold reliable representation in priority above aesthetics. There would certainly still be crosstalk. Art and science would continue to influence each other in design. However, a clear delineation of the goals, methods, and evaluation of the sonification would avoid design efforts that try to be both art and science and end up being neither.

\section{CONCLUSIONS}

Many of the challenges that faced early sonification researchers persist to the present day. Clearly outlining the goals of a given sonification, whether scientific or artistic, and holding fast to design principles and evaluations that best serve those goals may help us overcome some of these challenges. In a keynote address at ICAD in 2017, Carla Scaletti suggested that sonification may be near the tipping point of "scientific legitimacy" [34]. Sonification may also be at a tipping point of "artistic legitimacy." Decoupling these approaches may facilitate tipping points for sonification in both domains.

\section{REFERENCES}

[1] G. Kramer et al., "Sonification report: Status of the field and research agenda.," Palo Alto, CA:, 1999.

[2] M. A. Nees, "Auditory Graphs Are Not the "Killer App" of Sonification, But They Work," Ergonomics in Design, vol. 26, no. 4, pp. 25-28, Oct 2018, doi: $10.1177 / 1064804618773563$.

[3] A. Supper, "The Search for the "Killer Application": Drawing the Boundaries around the Sonification of Scientific Data," in The Oxford Handbook of Sound Studies, T. Pinch and K. Bijsterveld Eds. New York: Oxford University Press, 2012, pp. 249-270.

[4] J. H. Flowers, "Thirteen years of reflection on auditory graphing: Promises, pitfalls, and potential new directions," presented at the International Conference on Auditory Display, Limerick, Ireland, 2005.

[5] B. N. Walker and M. A. Nees, "Theory of Sonification," in The Sonification Handbook, T. Hermann, A. Hunt, and J. G. Neuhoff Eds. Berlin: Logos Publishing House, 2011.

[6] T. Hermann, A. Hunt, and J. G. Neuhoff, "Introduction," in The Sonification Handbook, T. Hermann, A. Hunt, and J. G. Neuhoff Eds. Berlin: Logos Publishing House, 2011, pp. 1-6.

[7] M. Ballora, "Sonification, Science and Popular Music: In search of the 'wow'," Organised Sound, vol. 19, no. 1, pp. 30-40, Apr 2014, doi: 10.1017/s1355771813000381.

[8] A. Supper, "Lobbying for the Ear: The Public Fascination with and Academic Legitimacy of the Sonification of Scientific Data," Ph. D., Maastricht University, 2012.

[9] A. Supper, "Sublime frequencies: The construction of sublime listening experiences in the sonification of scientific data," Social Studies of Science, vol. 44, no. 1, pp. 34-58, Feb 2014, doi: 10.1177/0306312713496875.

[10] G. Dubus and R. Bresin, "A Systematic Review of Mapping Strategies for the Sonification of Physical Quantities,", Plos One, Article vol. 8, no. 12, p. 28, Dec 2013, Art no. e82491, doi: 10.1371/journal.pone.0082491.

[11] H. Ono, "Difference threshold for stimulus length under simultaneous and nonsimultaneous viewing conditions," Perception \& Psychophysics, vol. 2, no. 5, pp. 201-207, 1967, doi: 10.3758/bf03213050.

[12] R. Teghtsoonian, "Exponents in stevens law and constant in ekmans law," Psychological Review, Article vol. 78, no. 1, pp. 71-+, 1971, doi: 10.1037/h0030300.

[13] D. R. Perrott and K. Saberi, "Minimum audible angle thresholds for sources varying in both elevation and azimuth," Journal of the Acoustical Society of America, vol. 87, no. 4, pp. 1728-1731, Apr 1990, doi: 10.1121/1.399421.

[14] A. Shaqiri et al., "Sex-related differences in vision are heterogeneous," Scientific Reports, vol. 8, May 2018, Art no. 7521 , doi: $10.1038 / \mathrm{s} 41598-018-25298-8$.

[15] B. N. Walker, "Magnitude estimation of conceptual data dimensions for use in sonification," Journal of Experimental Psychology-Applied, Article; Proceedings 
Paper vol. 8, no. 4, pp. 211-221, Dec 2002, doi: 10.1037/1076-898x/8.4.211.

[16] L. Axon, M. Goldsmith, and S. Creese, "Sonification Mappings: Estimating Effectiveness, Polarities, and Scaling in an Online Experiment," Journal of the Audio Engineering Society, Article vol. 66, no. 12, pp. 10161032, Dec 2018, doi: 10.17743/jaes.2018.00057.

[17] J. G. Neuhoff, R. Knight, and J. Wayand, "Pitch change, sonification, and musical expertise: Which way is up?," presented at the International Conference on Auditory Display, Kyoto, Japan, 2002.

[18] L. Kishon-Rabin, O. Amir, Y. Vexler, and Y. Zaltz, "Pitch discrimination: Are professional musicians better than non-musicians?," Journal of Basic and Clinical Physiology and Pharmacology, Article vol. 12, no. 2, pp. 125-143, 2001.

[19] C. Marie, T. Kujala, and M. Besson, "Musical and linguistic expertise influence pre-attentive and attentive processing of non-speech sounds," Cortex, Article vol. 48, no. 4, pp. 447-457, Apr 2012, doi: 10.1016/j.cortex.2010.11.006.

[20] P. Janata and K. Paroo, "Acuity of auditory images in pitch and time," Perception \& Psychophysics, vol. 68 no. 5, pp. 829-844, Jul 2006, doi: 10.3758/bf03193705.

[21] B. R. Zendel and C. Alain, "Concurrent Sound Segregation Is Enhanced in Musicians," Journal of Cognitive Neuroscience, Article vol. 21, no. 8, pp. 14881498, Aug 2009, doi: 10.1162/jocn.2009.21140.

[22] J. G. Neuhoff, "Interacting Perceptual Dimensions," in Ecological Psychoacoustics, J. G. Neuhoff Ed. New York: Academic Press, 2004.

[23] R. D. Melara and L. E. Marks, "Interaction among auditory dimensions - timbre, pitch, and loudness," Perception \& Psychophysics, Article vol. 48, no. 2, pp. 169-178, Aug 1990, doi: 10.3758/bf03207084.

[24] J. G. Neuhoff, "Perceptual bias for rising tones," Nature, Letter vol. 395, no. 6698, pp. 123-124, SEP 101998 1998, doi: 10.1038/25862.

[25] C. C. Wang, "Effects of some aspects of rhythm on tempo perception," Journal of Research in Music Education, vol. 32, no. 3, pp. 169-176, 1984, doi: $10.2307 / 3344836$.

[26] P. G. Vos, M. vanAssen, and M. Franek, "Perceived tempo change is dependent on base tempo and direction of change: Evidence for a generalized version of Schulze's (1978) internal beat model," Psychological Research-Psychologische Forschung, Article vol. 59, no. 4, pp. 240-247, Feb 1997, doi: 10.1007/bf00439301.

[27] C. Wollner, J. Ginsborg, and A. Williamon, "Music researchers' musical engagement," Psychology of Music, Article vol. 39, no. 3, pp. 364-382, Jul 2011, doi: $10.1177 / 0305735610381592$.

[28] S. E. Pitts, "Amateurs as Audiences: Reciprocal Relationships between Playing and Listening to Music," in Audience Experience: a Critical Analysis of Audiences in the Performing Arts, J. Radbourne, H. Glow, and K. Johanson Eds. Oxford: Intellect Ltd, 2013, pp. 83-+.

[29] B. P. Gold, M. J. Frank, B. Bogert, and E. Brattico, "Pleasurable music affects reinforcement learning according to the listener," Frontiers in Psychology, Article vol. 4, p. 19, Aug 2013, Art no. 541, doi: 10.3389/fpsyg.2013.00541.

[30] A. Harris and E. Flynn, "Medical education of attention: A qualitative study of learning to listen to sound," Medical Teacher, vol. 39, no. 1, pp. 79-84, Jan 2017, doi: 10.1080/0142159x.2016.1231916.
[31] L. M. Mauney and B. N. Walker, "Individual Differences and the Field of Auditory Display: Past Research, A Present Study, and an Agenda for the Future," presented at the International Conference on Auditory Display, Montréal, 2007.

[32] T. L. Bonebright and J. H. Flowers, "Evaluation of auditory display," in The Sonification Handbook, T. Hermann, A. Hunt, and J. G. Neuhoff Eds., 2011, pp. 111-144.

[33] S. Landry and M. Jeon, "Participatory Design Research Methodologies: A Case Study In Dancer Sonification," presented at the The 23rd International Conference on Auditory Display, Pennsylvania State University, 2017.

[34] C. Scaletti, "Why sonification is a joke. Keynote address delivered at the 23rd International Conference on Auditory Display,," ed. University Park, PA., 2017, p. https://www.youtube.com/watch?v=T0qdKXwRsyM.

[35] S. Barrass, "The aesthetic turn in sonification towards a social and cultural medium," AI \& Society, vol. 27, pp. 177-181, 2012, doi: 10.1007/s00146-011-0335-5.

[36] S. Barrass, M. Whitelaw, and F. Bailes, "Listening to the mind listening: An analysis of sonification reviews, designs and correspondences," Leonardo Music Journal, Article vol. 16, pp. 13-19, 2006, doi: 10.1162/lmj.2006.16.13.

[37] J. Neuhoff and M. McBeath, "The Doppler illusion: The influence of dynamic intensity change on perceived pitch," Journal of Experimental Psychology-Human Perception and Performance, vol. 22, no. 4, pp. 970-985, AUG 1996 1996, doi: 10.1037/0096-1523.22.4.970.

[38] M. G. Boltz, "Illusory tempo changes due to musical characteristics," Music Perception, vol. 28, no. 4, pp. 367-386, Apr 2011, doi: 10.1525/mp.2011.28.4.367.

[39] D. E. MacDonald, T. Natarajan, and R. C. Windeyer, "Data-driven sonification of cfd aneurysm models," presented at the International Conference on Auditory Display, Michigan Technological University, 2018.

[40] M. Ballora, C. Roman, R. Pockalny, and K. Wishner, "Sonification and science pedagogy: Preliminary experiences and assessments of earth science data presented in an undergraduate general education course," presented at the International Conference on Auditory Display, Michigan Technological University, 2018.

[41] R. Wheeler and D. Worrall, "Representing twitter users' engagement by sonification," presented at the International Conference on Auditory Display, Michigan Technological University, 2018.

[42] W. W. Gaver, "What in the world do we hear? An ecological approach to auditory event perception," Ecological Psychology, 5(1), 1-29. 1993, pp. 1-29.

[43] J. G. Neuhoff and L. M. Heller, "One small step: Sound sources and events as the basis for auditory graphs," in 11th International Conference on Auditory Display, Limerick, Ireland, 2005.

[44] S. C. Provost. "Communicating with Non-Musicians." https://sarahcprovost.domains.unf.edu/communicatingwith-non-musicians/ (accessed April 2, 2019, 2019).

[45] M. Gallant. "Collaborating and communicating with nonmusicians."

https://blog.discmakers.com/2018/07/collaborating-andcommunicating-with-non-musicians/ (accessed April 2, 2019, 2019). 